\title{
Therapeutic outcome of diverticular associated colitis - a retrospective single- centre experience
}

\author{
F. Vulsteke', G. De Hertogh', S. Vermeire ${ }^{3}$ \\ (1) Department of Internal Medicine, University Hospitals Leuven, Leuven, Belgium ; (2) Department of Pathology, University Hospitals Leuven, Leuven, Belgium ; \\ (3) Department of Gastroenterology \& Hepatology, University Hospitals Leuven, Leuven, Belgium.
}

\begin{abstract}
Background : Diverticular associated colitis (DAC) has become increasingly appreciated as a form of inflammatory disease, localized mainly in the sigmoid, and defined as chronic inflammation of the interdiverticular mucosa with sparing of rectum, right colon and diverticula themselves.

Methods : A retrospective case identification from January 2005 to December 2016 was performed. Patients with a diagnosis of DAC based on clinical, endoscopic and histological findings were enrolled. We analyzed their characteristics and response to therapy, and performed a review of literature.

Results : Out of 377 pathology reports, 37 cases of DAC were identified, with a median age of 73 years and followed during 1-13 years. Six patients $(16.22 \%)$ were refractory to conservative treatment and required surgery. In three patients $(8.11 \%)$ evolution to ulcerative colitis (UC) was observed. Patients were divided into four endoscopic patterns, with a more benign course for type A "crescentic fold disease" compared to the other subtypes. Patients with type B "mild to moderate ulcerative colitis-like" were at significantly higher risk of persistent disease activity or relapse (p<0.01).

Conclusion : DAC is a multifaceted disease and considered to be a relatively benign condition. However, a subset of patients requires surgery and/or may progress to develop UC. (Acta gastroenterol. belg., 2021, 84, 275-281).
\end{abstract}

Key words : segmental colitis, diverticulosis, inflammatory bowel disease.

\section{Background}

Diverticular associated colitis (DAC), also known as diverticular disease-associated segmental colitis, or segmental colitis associated with diverticulosis, is a newly established disorder of chronic inflammation in the colon harbouring diverticula, mostly located in the sigmoid region. The reported prevalence ranges from $0.25 \%$ to $1.4 \%$ in the general population, and $1.15 \%$ to $11.4 \%$ amongst those suffering from diverticular disease (1). By definition, both the rectum and the right colon are spared from any inflammation both endoscopically and histologically (2). The inflammatory process originates from the interdiverticular mucosa with sparing of the diverticular orifices as well, and differs in that way from diverticulitis in which inflammation is observed only inside and around diverticula. The pathogenesis is not completely understood and most likely multifactorial. DAC tends to develop almost exclusively in older adults, and predominantly, but not exclusively, in males. It most often presents with (painless) rectal bleeding, diarrhoea and/or abdominal pain. DAC pertains to the spectrum of inflammatory bowel diseases (IBD) and as such may be difficult to differentiate both macroscopically and histologically from Crohn's disease (CD) or ulcerative colitis (UC). However, in general, DAC generally responds to drug therapy and usually has a more benign clinical course. Only a small subset progresses to classical IBD (3). Currently there are no guidelines for its management, which is usually based on the administration of 5-aminosalicylates and antibiotics, with surgery being reserved for refractory cases. Not infrequently, patients with DAC have an entirely self-limited clinical course.

Data on DAC is scarce and mostly retrospective, with a lack of uniformity regarding the precise diagnostic criteria. We therefore retrospectively identified all patients diagnosed with DAC at our tertiary IBD referral centre at the University Hospitals Leuven, Belgium, to better define the clinical, endoscopic, and histological characteristics that allow proper identification and diagnosis of DAC, as well as available treatments and outcome.

\section{Material and methods}

\section{Patient selection}

A retrospective analysis was performed of all patients who were diagnosed with DAC at the University Hospitals Leuven Belgium. For this, an automated search was done via the electronic medical record system of the pathology reports of all colon biopsies obtained between January 2005 to December 2016. The following search terms were used: "diverticular associated colitis", "segmental colitis", "segmental colitis associated with diverticular disease". Final diagnosis of DAC was established by the combination of endoscopic and histopathological features. The endoscopic criteria for inclusion included erythema, granularity and friability within the region of the colon harbouring diverticula, sparing of the diverticular orifices, endoscopic sparing of the rectum and proximal colon (4). Therefore, both the endoscopic descriptions as

Correspondence to: S. Vermeire, Department of Gastroenterology and Hepatology University Hospitals Leuven, KU Leuven, Herestraat 49 B-3000 Leuven, Belgium. Phone : + 32163442 18. Fax : + 3216344419.

Email : Severine.Vermeire@uzleuven.be

Submission date : 10/11/2019

Acceptance date : 22/08/2020 
the images made during colonoscopies were evaluated. The remaining patients were subsequently reviewed for demographics, symptoms, history and use of medications (antibiotics as well as non-steroidal anti-inflammatory drugs (NSAIDs)), results of stool cultures, laboratory examinations and treatment. The protocol of this study was approved by the ethics committee of the Medical Faculty of the University of Leuven on September 26, 2017 (MP001996).

\section{Statistical assessment}

Continuous variables are expressed as medians with interquartile range. Differences between proportions were assessed using Chi Square test or Fisher's exact test where appropriate. The level for significance was set at $\mathrm{p}<0.05$.

\section{Study selection}

A literature search from January 2000 to February 2018 was conducted by use of Medline, EMBASE and Cochrane databases. PubMed and Ovid were used as search engines. The following medical subject heading (MeSH) terms were used without language restriction: "diverticular associated colitis," "segmental colitis," "segmental colitis associated with diverticular disease", "diverticulosis", "diverticulitis", "inflammatory bowel disease" and "colitis." Additional studies cited within the

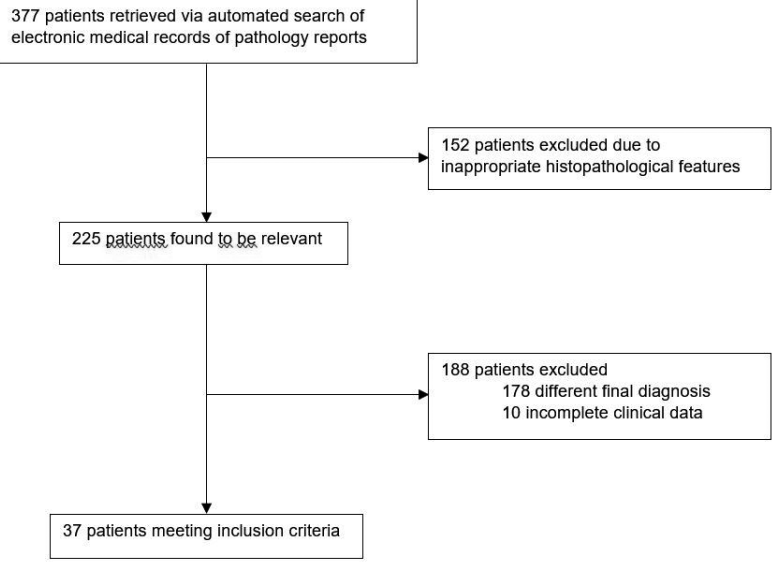

Figure 1. - Flow chart of study inclusion and exclusion criteria.

literature were also searched. Boolean operators ("and", "or", "not") were used to narrow and widen the search.

\section{Results}

\section{Patients}

The automated search of the pathology reports of colon biopsies resulted in 377 patients, of whom 152 had inadequate arguments for DAC based on the

Table 1. - Baseline characteristics of patients with diverticular associated colitis $(\mathbf{n}=\mathbf{3 7})$

\begin{tabular}{|l|c|}
\hline CHARACTERISTIC & NO. OF PATIENTS (\%) \\
\hline Median age of onset (range) & $73(50-92)$ \\
\hline Gender & \\
$\quad$ Male & $21(56.76)$ \\
$\quad$ Female & $16(43.24)$ \\
\hline Overweight (BMI > 25kg/m $\left.{ }^{2}\right)$ & $13(35.14)$ \\
\hline Smoker & $13(35.14)$ \\
$\quad$ Ex-smoker at diagnosis & $12(32.43)$ \\
$\quad$ Active smoker at diagnosis & $1(2.70)$ \\
\hline Familial history of IBD & $3(8.11)$ \\
\hline History of acute diverticulitis & $7(18.92)$ \\
\hline Signs and symptoms & \\
Asymptomatic & $3(8.11)$ \\
> 1 symptom at diagnosis & $21(56.76)$ \\
Haematochezia & $28(75.68)$ \\
Diarrhoea & $9(24.32)$ \\
Bowel movement disturbance & $16(43.24)$ \\
Hemodynamic instable & $2(5.41)$ \\
Mucoid stools & $11(29.73)$ \\
Flatus & $4(10.81)$ \\
Tenesmus & $4(10.81)$ \\
Abdominal pain & $13(35.14)$ \\
Weight loss & $9(24.32)$ \\
Fatigue & $1(2.70)$ \\
Anorexia & $2(5.41)$ \\
Pyoderma gangrenosum & $1(2.70)$ \\
\hline
\end{tabular}

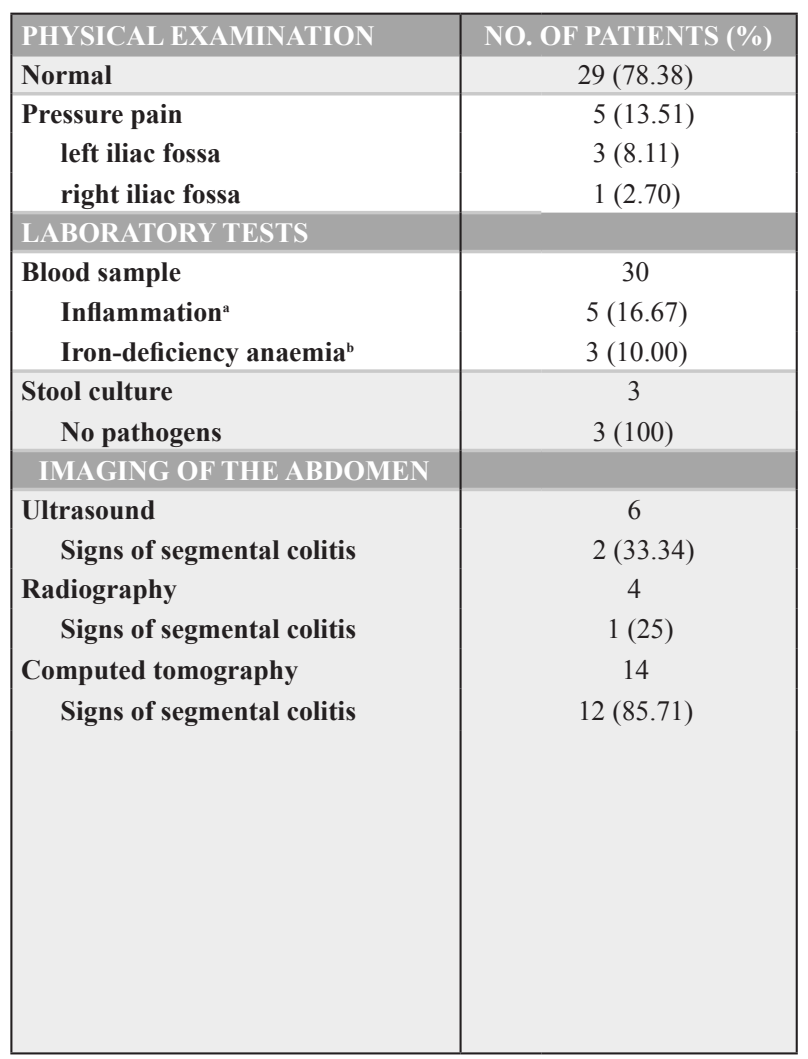

${ }^{a}$ Inflammation based on C-reactive protein and leucocytosis. ${ }^{b}$ Iron-deficiency anaemia was statistically significant for persistent symptoms $(p=0.042)$. There were no significant differences in outcome for any of the other characteristics (Fisher's exact testing, $p<0.05$ ). 
Table 2. - All patients $(\mathbf{n}=\mathbf{3 7})$ subdivided according to endoscopic subtypes of diverticular associated colitis

\begin{tabular}{|c|c|c|c|c|}
\hline Pattern & A-Crescentic fold & B - Mild-to-moderate UC-like & $\mathrm{C}$-CD-like & D - Severe UC-like \\
\hline No. of patients $(\%)$ & $17(45.95)$ & $15(40.54)$ & $1(2.70)$ & $4(10.81)$ \\
\hline $\begin{array}{l}\text { Endoscopic } \\
\text { appearance }\end{array}$ & $\begin{array}{l}\text { Reddish round lesions } \\
\text { from } 0.5 \text { to } 1.5 \mathrm{~cm} \text { in } \\
\text { diameter at the top of } \\
\text { the colonic folds }\end{array}$ & $\begin{array}{l}\text { Loss of the submucosal } \\
\text { vascular pattern, } \\
\text { oedema, hyperaemia, } \\
\text { diffuse erosions }\end{array}$ & $\begin{array}{l}\text { Isolated aphthous } \\
\text { ulcers }\end{array}$ & $\begin{array}{l}\text { Loss of the submucosal } \\
\text { vascular pattern, oedema } \\
\text { with reduced calibre of } \\
\text { colonic lumen, intense } \\
\text { hyperaemia, diffuse } \\
\text { ulcerations }\end{array}$ \\
\hline $\begin{array}{l}\text { Sparing of rectum } \\
\text { and diverticular } \\
\text { orifices }\end{array}$ & Yes & Yes & Yes & Yes \\
\hline $\begin{array}{l}\text { Histological } \\
\text { features }\end{array}$ & $\begin{array}{l}\text { No significant distortion } \\
\text { of the glandular } \\
\text { architecture }\end{array}$ & $\begin{array}{l}\text { Distortion of crypt } \\
\text { architecture, chronic } \\
\text { changes in lamina } \\
\text { propria }\end{array}$ & $\begin{array}{l}\text { Highest variability. } \\
\text { Transmural } \\
\text { inflammation with } \\
\text { microfissures }\end{array}$ & $\begin{array}{l}\text { Distortion of crypt } \\
\text { architecture, crypt } \\
\text { haemorrhage, chronic } \\
\text { changes in lamina propria }\end{array}$ \\
\hline Cellular features & $\begin{array}{l}\text { Neutrophil and } \\
\text { lymphocyte infiltrates: } \\
\text { unorganized and limited } \\
\text { to crypt epithelium }\end{array}$ & $\begin{array}{l}\text { Crypt abscesses and } \\
\text { goblet cell depletion }\end{array}$ & $\begin{array}{l}\text { epithelioid granulomas, } \\
\text { and non-specific } \\
\text { infiltrates }\end{array}$ & $\begin{array}{l}\text { Crypt abscesses and } \\
\text { goblet cell depletion }\end{array}$ \\
\hline
\end{tabular}

UC, ulcerative colitis ; CD, Crohn's disease.

histopathological pattern alone. Clinical and endoscopic findings were retrieved from the remaining 225 patients. Another 178 patients were excluded due to a final differential diagnosis based on combined endoscopic and histopathological patterns. Ten patients were excluded because of incomplete clinical data (Figure 1). We finally included 37 patients, of whom 21 male and 16 female Caucasians. Nineteen patients had confirmed rectal sparing on biopsy.

The clinical characteristics of the patients are presented in Table 1. The median age was 73 years (range 50-92 years) with a duration of follow-up ranging from 1 to 13 years (median duration of 5.75 years). Thirteen (35.14\%) patients had a history of smoking, while only one patient $(2.70 \%)$ was actively smoking at time of diagnosis. Other known cardiovascular risk factors are shown in table 1 . No patient took NSAIDs at time of diagnosis. Prior to diagnosis, fourteen patients (37.84\%) were already known with diverticulosis, and half of them had a history of acute diverticulitis at least 2 years earlier. Three patients progressed to UC after a median followup of 11 months.

\section{Signs and symptoms}

The most recurrent symptom at presentation, present in 28 patients $(75.68 \%)$ was haematochezia. Two patients $(5.42 \%)$ were even haemodynamically unstable due to excessive rectal bleeding. Bowel movement disturbance was observed in 16 patients $(43.24 \%)$, diarrhoea in $24.32 \%$ of patients, and mucoid stools in $29.73 \%$ of patients. Abdominal pain was present in 13 patients (35.14\%), four of whom presented with associated tenesmus $(10.81 \%)$. Less frequent symptoms included weight loss, flatulence, anorexia and distinct fatigue in respectively nine $(24.32 \%)$, four $(10.81 \%)$, two $(5.41 \%)$ and one $(2.70 \%)$ of the patients. The duration of symptoms before seeking medical advice ranged from 1 day to 35 years, with a median duration of 2.5 months. Interestingly, three patients $(8.11 \%)$ were completely asymptomatic and DAC was an accidental finding during routine colonoscopy for cancer screening. None of the patients had perianal disease, nor colorectal cancer during follow-up.

Laboratory results, performed in 30 of 37 patients, showed no signs of inflammation in $66.67 \%(25 / 30)$. Three patients (10\%) had iron-deficiency anaemia, which was a statistically significant sign to predict persistent symptoms $(\mathrm{p}=0.042)$.

\section{Endoscopic and histologic findings}

Endoscopic abnormalities were restricted to the left colon, with rectal-, transverse- and right colonsparing features. One patient had extension of colonic inflammation into the descending colon. The endoscopic features of diverticular colitis were classified into four patterns, based on prior descriptions in literature $(1,5)$. Sparing of both rectum and diverticular orifices is the unifying feature between the four subtypes (Table 2).

Most patients (46\%) had type A "crescentic fold pattern", characterized by reddish round lesions ranging from 0.5 to $1.5 \mathrm{~cm}$ in diameter at the top of the colonic folds, with no ulcers or signs of bleeding. Secondly, $41 \%$ of patients had type B "mild-to-moderate ulcerative colitis-like pattern", which is similar to mild to moderate UC. Reported features were mucosal oedema, loss of submucosa vascular system, hyperaemia and diffuse erosions. In our cohort only one patient $(2.70 \%)$ had type C "Crohn-colitis-like pattern" with isolated linear ulcers, 




Figure 2. - Endoscopic classification of diverticular associated colitis, with corresponding histopathological features. Images are obtained from the electronic medical record system of the University Hospitals of Leuven. The histopathological images are reviewed by an IBD-specialised pathologist. A1. 'Crescentic fold disease'. The colonic mucosa shows swollen red patches of 0.5 to $1.5 \mathrm{~cm}$ in diameter confined to the crescentic mucosal fold, without haemorrhage or ulceration. A2. Chronic colitis with discrete architectural defects (rare branching crypts), patchy increased infiltrate and well-preserved epithelium (25x OM). B1. 'Mild-to-moderate ulcerative colitis-like'. The colonic mucosa shows loss of vascular pattern, oedema and hyperaemia of the mucosa with diffuse erosions. B2. Active inflammation with cryptitis (200x OM). C1. 'Crohn's disease colitis-like'. The endoscopic pattern shows scattered linear ulcers within a normal colonic mucosa. C2. Disturbed architecture (cryptal abnormalities and irregular mucosal surface), diffuse mixed inflammatory cell infiltrates (25x OM). D1. 'Severe ulcerative colitis-like'. The mucosa shows a marked oedema with ulcerations, reduction of lumen, diffuse loss of vascular pattern, diffuse hyperaemia and easy contact bleeding. D2. Seriously disturbed architecture with erosions, cryptabcesses, basal plasmocytosis and eosinophils (200x OM).

but a mucosa of normal endoscopic appearance, which is very similar to mild to moderate $\mathrm{CD}$. The remaining four patients $(11 \%)$ had type D "severe ulcerative colitis-like pattern" which resembles severe UC, with loss of submucosa pattern, intense hyperaemia, diffuse ulcerations and reduced calibre of colic lumen (Table 2). (Figure 2).

The histopathological features of all 37 patients were related to the endoscopic pattern as shown in Table 2 . Compared to the other types, type A ran a more benign course $(\mathrm{p}=0.0498)$, whereas patients with DAC type B were at significant higher risk of persistent disease or relapse $(\mathrm{p}=0.0069)$.

\section{Treatment and outcome}

Most patients (73\%) received anti-inflammatory treatment, while the remaining $27 \%$ were advised only antispasmodics or became spontaneously asymptomatic. Antibiotics were first-line treatment in twelve patients 
Table 3. - Outcome of patients with diverticular associated colitis based on treatment during the follow-up period

\begin{tabular}{|c|c|c|c|c|}
\hline \multirow[t]{2}{*}{ Patients with DAC $(\mathrm{n}=37)$} & \multirow{2}{*}{$\begin{array}{l}\text { NO. OF PATIENTS } \\
\text { ( } \% \text { of all patients) }\end{array}$} & \multicolumn{3}{|c|}{ OUTCOME ( $\%$ of patients with corresponding treatment) } \\
\hline & & Resolution & Still on active treatment & $\begin{array}{l}\text { Relapse after symptom- and } \\
\text { treatment-free interval }\end{array}$ \\
\hline No or only supportive treatment & $10(27.03)$ & NA & NA & NA \\
\hline Any anti-inflammatory treatment & $27(72.97)$ & $22(81.48)$ & $10(37.04)$ & $2(7.41)$ \\
\hline Antibiotics & $12(32.43)$ & $1(8.33)$ & $0(0)$ & $0(0)$ \\
\hline Metronidazole $500 \mathrm{mg}$ t.i.d. & $9(24.32)$ & $1(11.11)$ & & \\
\hline +Ciprofloxacin $500 \mathrm{mg}$ b.i.d. & $3(8.11)$ & $0(0)$ & & \\
\hline 5-ASA monotherapy & $23(62.16)$ & & & \\
\hline Rectal & $6(16.22)$ & $2(33.33)$ & $0(0)$ & $1(16.67)$ \\
\hline Oral & $17(45.95)$ & $15(88.24)$ & $6(35.29)$ & $1(5.88)$ \\
\hline Steroids monotherapy & $4(10.81)$ & & & \\
\hline Rectal & $0(0)$ & NA & NA & NA \\
\hline Oral & $4(10.81)$ & $2(50)$ & $1(25)$ & $0(0)$ \\
\hline 5-ASA and steroids & $10(27.03)$ & $8(80)$ & $1(10)$ & $0(0)$ \\
\hline Antimetabolites (MTX, azathioprine) & $(25.41)$ & $2(100)$ & $2(100)$ & NA \\
\hline Sigmoid colectomy & $6(16.22)$ & $6(100)$ & NA & $0(0)$ \\
\hline
\end{tabular}

*Resolution is defined as the absence of clinical symptoms. T.i.d., three times a day; b.i.d., twice a day; 5-ASA, 5-aminosalicylic acid; NA, not applicable.

$(32.43 \%)$, however, only one patient responded. Twentythree patients $(62.16 \%)$ received 5-aminosalicylic acid (5-ASA) monotherapy in varying doses (1 to $3 \mathrm{~g} /$ day), with a success rate of $88 \%$ when administered orally. Ten patients $(27.03 \%)$ required additional use of steroids (budesonide or beclomethasone dipropionate mostly oral) to induce remission. Remission was obtained in $80 \%$. Two and six patients, refractory to conventional treatments, received respectively immunomodulator therapy (methotrexate or azathioprine) or underwent surgery (sigmoid resection). No postoperative recurrence was seen in any of these six patients in the years of follow-up. No patients were administered biologicals or probiotics. Of all patients with DAC, only ten patients $(27.03 \%)$ needed maintenance therapy, so over $70 \%$ of patients maintained in clinical remission for years without any therapy (Table 3).

\section{Discussion}

The concept of DAC as a disease was established by Ludeman and Stepherd in 2002 in a study of 98 patients (6). As most studies are retrospective and given the overlap with other conditions, the real prevalence of the disease may be underestimated. Admittedly, the endoscopic signs and histologic features may be diverse and non-specific, rendering accurate diagnosis difficult (6). Moreover, the entity is almost exclusively a disorder of the elderly, with an observed median age at diagnosis in our series of 73 years and a minimum age of 50 . These figures are in line with those reported by others $(7,8)$. We also report a slightly higher male preponderance of $56.76 \%$, which is also in line with a recent meta-analysis (9).

The clinical presentation of DAC is highly variable, and patients can present with a range of symptoms. In our study rectal bleeding was the most frequent symptom, present in over three quarters of patients, followed by abdominal pain, mucoid stools and diarrhoea. Systemic features, such as weight loss, fatigue and fever were rare, in line with other retrospective studies $(2,6,7)$. The duration of symptoms differed widely from 1 day to 35 years $(6,10)$. One patient suffered also from pyoderma gangrenosum. The association of DAC with extraintestinal manifestations (e.g. arthritis, ankylosing spondylitis, erythema nodosum, and pyoderma gangrenosum) has been described, but remains largely unknown (7). None of our patients took NSAIDs at time of diagnosis, which is important to rule out medicationassociated colitis.

Although no significant differences in symptoms were found depending on the endoscopic subtype, diarrhoea is reported more common in type A, whereas patients with type $\mathrm{C}$ and $\mathrm{D}$ are more likely to experience rectal bleeding (1). In one of the few prospective studies on DAC by Tursi et al., there was no significant association between symptoms and endoscopic findings (5). Of the four categories, type A had the most benign course $(\mathrm{p}<0.05)$, whereas patients with DAC type B were at significant higher risk of persistent disease or relapse $(p$ $<0.01$ ). Because too few patients were diagnosed with type $\mathrm{C}$ and $\mathrm{D}$, we cannot make any firm statements on these subgroups. Tursi et al. reported that patients with DAC type B or D are at significant higher risk for a relapsing and remitting course. Hence, medical treatment in the latter two subtypes should be strongly considered (11). Laboratory investigations were completely normal in the majority of our patients, with inflammation and iron-deficiency anaemia in only a minority. However, iron-deficiency anaemia is a significant predictor for a more serious course of the disease. Stool culture and imaging studies were only performed in cases to rule out differential diagnoses as infectious enteritis, ischemic colitis or malignancy. 
The exact pathogenesis of DAC remains unclear, but is most likely multifactorial. Diverticulosis in itself is accompanied by muscle hypertrophy and bowel shortening, which lead to mucosal redundancy (12). Pressure and shear stress applied to the excessive mucosa lead to polypoid lesions and inflammatory changes characteristic of mucosal prolapse. Another mechanism is relative ischaemia due to age and other cardiovascular risk factors. Furthermore, faecal stasis that occurs in diverticular segments has been linked to the pathogenesis of DAC, as it is also an underlying mechanism of UC where dysbiosis can trigger the immune response in the susceptible host. Associated with faecal stasis, the increased exposure to intraluminal antigens and toxins, and changes in bacterial flora and bacterial enzyme activity are hypothesized to cause chronic mucosal inflammation $(1,6)$.

Whether DAC exists as a separate entity, or belongs to the spectrum of IBD, is still unclear. Not only the clinical presentation of DAC is indistinguishable from IBD, DAC can closely mimic the histological changes of UC or CD as well. Patients affected by DAC present much higher levels of tumour necrosis factor-alpha (TNF- $\alpha$ ), compared to a control population, which could support the hypothesis that DAC should be considered as part of the IBD spectrum (2). One case report noted an excellent response to infliximab (infusions of $5 \mathrm{mg} / \mathrm{kg}$ IV at 0,2 and 6 weeks, followed by maintenance infusions every 8 weeks) with a reduction of TNF- $\alpha$ levels and induction of remission (13). Additionally, $\mathrm{UC}$ with relative rectal sparing has become a recognized entity, though uncommon (14). In three patients $(8.11 \%)$ we reported the progression of DAC to conventional UC with rectal involvement after a follow-up period of 9 to 18 months. Two of them had a confirmed completely normal rectal biopsy. This unusual progression has only been described in case reports, with a mean progression time of 11 months, and remains difficult to predict (7). Thus far, it is not clear if the co-existence is due to a milieu prone for the development of UC through faecal stasis and bacterial mucolysis rather than purely circumstantial $(3,6)$.

Older patients diagnosed with CD are more likely to suffer from distal colitis and proctitis rather than small bowel and proximal large bowel involvement seen in younger patients (15). All three pathological hallmarks of $\mathrm{CD}$, i.e., transmural inflammation, epithelioid cell granulomas and fissuring or fistula formation, can in fact be seen as a result of diverticular disease alone. However, to make the distinction in individuals suspected of having both $\mathrm{CD}$ and diverticular disease, the remainder of the gastrointestinal tract should be investigated for evidence of CD elsewhere (i.e. small intestine, stomach).

None of our patients developed colorectal adenocarcinoma during follow-up. In contrast to IBD, there is little evidence that patients with $\mathrm{DAC}$ are at higher risk of colonic neoplasia (16).

The endoscopic appearance of DAC can vary in severity and is crucial to establish a correct diagnosis.
Based on endoscopic features, DAC has been classified into four different subtypes $(1,5)$. In our study, histological changes correlated well with the different macroscopic findings (Table 2). Tursi et al. postulated that different mechanisms underpin the changes seen in the different subtypes (5).

The histopathological findings of the affected sigmoid biopsies were not pathognomonic and demonstrated difficulty in distinguishing DAC from other forms of segmental colitis, including $\mathrm{CD}, \mathrm{UC}$, ischemic colitis, medication-associated colitis, infectious colitis and even acute uncomplicated diverticulitis. Only the absence of endoscopic and histological inflammation in the rectum allows correct differential diagnosis with UC. However, only $51.35 \%$ of our patients had a rectal biopsy. Nevertheless, in none of these patients macroscopic signs of proctitis were observed. Patients with endoscopic type A often had lymphocyte and neutrophilic inflammatory infiltration with no significant alteration of the glandular architecture. Patients with an UC-like pattern of DAC (type $\mathrm{B}$ and $\mathrm{D}$ ) had chronic changes in the lamina propria, including nonspecific inflammatory infiltrates and basal lymphocytosis, reduction of goblet cells and Paneth cell metaplasia, distortion of crypt architecture, cryptitis, crypt abscesses and crypt haemorrhage similar to that in UC. Our findings were comparable with the findings of Schembri et al. who reported the highest intragroup histological variability in those with an UC-like pattern (1). Our only patient with CD-like pattern had a totally different histological and cellular appearance with active and chronic transmucosal inflammation and microfistulas of the mucosa. No epithelioid granulomas were seen, not even with CD68 (KP1) immunostaining. The latter can help to distinguish between DAC and CD, but it is increasingly recognized that histological changes in adult $\mathrm{CD}$ can vary greatly with a minority showing evidence of noncaseating granulomatous inflammation (1).

Almost one third of patients did not need antiinflammatory treatment. This conservative approach has proven to incite favourable results, as was affirmed by Lamps and Knapple (4). In the other patients, remission was achieved in $81 \%$ with medical treatment including antibiotics, rectal or oral 5-ASA, oral corticosteroids and/ or antimetabolites. Antibiotics were mostly ineffective whereas oral 5-ASA was effective in $88 \%$ of patients, which is in line with the reported success rates by Freeman in 2016 (16). Two patients suffered recurrent rectal bleeding and needed re-administration of 5-ASA. A significant proportion $(27 \%)$ of patients required additional use of steroids (budesonide or beclomethasone dipropionate, with high "first-pass" metabolism) mostly oral, to induce remission. Six patients $(16 \%)$ required surgery but none had postoperative recurrences. Finally, only ten patients $(37 \%)$ needed maintenance therapy. Here, our findings are also consistent with the retrospective study by Freeman, who asserted that most patients with DAC, if followed for many years, have been 
observed to recover, often resolving completely within a few weeks or months with no recurrence (16).

A recent stepwise treatment algorithm for DAC by Schembri et al. suggests antibiotics as first-line treatment, whether or not together with 5-ASA and a high-fibre diet (1). Based on our results we would propose oral administration of 5-ASA as first-line treatment, with or without a short course of topical steroids to induce remission. Surgery is warranted for cases refractory to medical treatment.

In conclusion, Diverticular associated colitis (DAC) is an inflammatory process that affects mucosa in segments that are also affected by diverticulosis. It is more common in males and usually presents in the sixth to seventh decade of life. The clinical presentation is non-specific. Its diagnosis is based on the correlation of endoscopic and histologic findings and can be divided into four patterns. First-line treatment of DAC includes anti-inflammatory medication, such as mesalamine (5-ASA), although in some cases add-on of steroids is required. Only rarely, surgery is needed for refractory patients. Further prospective, randomized studies should investigate whether or not mild forms of the disease (i.e. DAC type A and C) need maintenance treatment to prevent disease recurrence. Whether DAC exists as a separate entity or whether it belongs in a spectrum with IBD is still unclear. Increased awareness about the condition and better liaison between the endoscopist and pathologist would increase the frequency of establishing an accurate diagnosis.

\section{Acknowledgement}

This research received no specific grant from any funding agency in the public, commercial, or not-forprofit sectors.

\section{Conflict of interest statement}

S. Vermeire : Research grant : MSD, AbbVie, Takeda, Pfizer, J\&J ; Lecture fee : MSD, AbbVie, Takeda, Ferring, Centocor, Hospira, Pfizer, J\&J, Genentech/
Roche ; Consultancy : MSD, AbbVie, Takeda, Ferring, Centocor, Hospira, Pfizer, J\&J, Genentech/Roche, Celgene, Mundipharma, Celltrion, SecondGenome, Prometheus, Shire, Prodigest, Gilead, Galapagos. SV is a senior clinical investigator of the Research Foundation - Flanders (FWO).

\section{References}

1. SCHEMBRI J., BONELLO J., CHRISTODOULOU DK., KATSANOS KH., ELLUL P. Segmental colitis associated with diverticulosis: Is it the coexistence of colonic diverticulosis and inflammatory bowel disease? Annals of Gastroenterology, 2017, 30 : 257-61.

2. TURSI A. Segmental colitis associated with diverticulosis: Complication of diverticular disease or autonomous entity? Digestive Diseases and Sciences, $2011,56: 27-34$

3. MAESHIRO T., HOKAMA A., KINJO T., FUJITA J. Diverticular colitis of the ascending colon preceding the onset of ulcerative colitis. BMJ Case Rep, 2014.

4. LAMPS LW., KNAPPLE WL. Diverticular Disease-Associated Segmental Colitis. Clin. Gastroenterol. Hepatol., 2007, 5(1) : 27-31.

5. TURSI A., ELISEI W., BRANDIMARTE G., GIORGETTI GM., LECCA PG., DI CESARE L., et al. The endoscopic spectrum of segmental colitis associated with diverticulosis. Color Dis., 2010, 12(5) : 464-70.

6. LUDEMAN L., SHEPHERD NA. What is diverticular colitis? Pathology, 2002, 34(6) : 568-72.

7. MULHALL AM., MAHID SS., PETRAS RE., GALANDIUK S. Diverticular disease associated with inflammatory bowel disease-like colitis : A systematic review. Dis. Colon Rectum., 2009, 52(6) : 1072-9.

8. TURSI A., JOSEPH RE., STRECK P. Expanding applications: The potential usage of 5-aminosalicylic acid in diverticular disease. Dig. Dis. Sci., 2011, 56(11) : 3112-21.

9. MANN NS., HODA KK. Segmental colitis associated with diverticulosis : Systematic evaluation of 486 cases with meta-analysis. Hepatogastroenterology, 2012, 59(119) : 2119-21.

10. RAMPTON DS. Diverticular colitis: Diagnosis and management. Color Dis., 2001, 3(3) : 149-53.

11. TURSI A., ELISEI W., GIORGETTI GM., INCHINGOLO CD., NENNA R., PICCHIO M., et al. Segmental colitis associated with diverticulosis : A 5-year follow-up. Int. J. Colorectal. Dis., 2012, 27(2) : 179-85.

12. OZTURK O., KOKLU H., AKBAL E., AKSOY EK., ALTAN E., BASAR O., et al. Diverticular disease and posture during defecation: a prospective comparative study. Acta Gastroenterol. Belg, 2018, 81(4) : 490-495.

13. HASSAN C., ZULLO A., IERARDI E., BURATTINI O., DE FRANCESCO V., MORINI S. Tumour necrosis factor $\alpha$ downregulation and therapeutic response to infliximab in a case of segmental colitis associated with diverticula. Gut., 2006, 55 : 589-90.

14. ORDÁS I., ECKMANN L., TALAMINI M., BAUMGART DC., SANDBORN WJ. Ulcerative colitis. Lancet, 2012, 380 (9853) :1606-19.

15. QUEZADA SM., STEINBERGER EK., CROSS RK. Association of age at diagnosis and crohn's disease phenotype. Age Ageing, 2013, 42(1) :102-6.

16. FREEMAN HJ. Segmental colitis associated diverticulosis syndrome. World Journal of Gastroenterology, 2016, 22 : 8067-9. 